\title{
VARIACIÓN SOCIOFONÉTICA DEL ESPAÑOL DE CHILE: PERCEPCIONES SUBJETIVAS DE FUTUROS PROFESORES DEL SISTEMA ESCOLAR ${ }^{1}$ \\ SOCIOPHONETIC VARIATION OF SPANISH IN CHILE: SUBJECTIVE PERCEPTIONS OF FUTURE TEACHERS IN THE SCHOOL SYSTEM
}

\author{
Andrea Carrasco Lobos \\ Universidad Metropolitana de Ciencias de la Educación. \\ carrascolobos.andrea@gmail.com \\ Héctor Ortiz Lira \\ Universidad Metropolitana de Ciencias de la Educación. \\ hectorortizlira@gmail.com
}

\section{Resumen}

El presente trabajo, de tipo cuantitativo, aborda las valoraciones subjetivas que estudiantes de pedagogías otorgan a distintas realizaciones de /tr/, / $/ \bar{r} /, \mid \mathrm{t} / \mathrm{y} / \mathrm{j} /$.

La muestra estuvo constituida por una selección aleatoria de estudiantes de distintas pedagogías de una universidad estatal. Para la recolección de datos, se utilizó la técnica de pares falsos, aproximación indirecta que encubre parcialmente el fenómeno de estudio. Entre los resultados más relevantes, se observa una aceptación parcial del alófono [J], la aparente imperceptibilidad de la transformación de la vibrante múltiple [r] en fricativa [.] y la tendencia desaprobatoria de aquellas realizaciones polémicas seguidas de vocales.

Como conclusión, se plantea la necesidad de estudiar los fenómenos fonéticos considerando factores contextuales, ideológicos y sociohistóricos, en tanto que su significado se construye en medio de la actividad social.

Palabras clave: Valoraciones subjetivas; Prestigio lingüístico; Estigmatización lingüística.

\begin{abstract}
This quantitative study analyzed the subjective evaluations of students of teacher-training programmes about different allophones of $/ \mathrm{tr} /,|\bar{r} /,| \mathrm{t} /$ and $/ \mathrm{j} /$.

The sample was comprised of a random selection of students from different teachertraining programmes from a Chilean state university. The matched-guise technique was adopted for data collection in order to partially conceal the phenomenon under study.

Among the most relevant findings, partial acceptance of the allophone [J] is reported, as well as an apparent imperceptibility of the substitution of trill [r] for fricative [1], and a tendency towards the disapproval of controversial allophones followed by vowels.

In conclusion, it is suggested that phonetic phenomena should be explored taking into consideration contextual, ideological and socio-historical factors, as they build their meaning in the middle of social activity.

Keyword: Subjective evaluations; Linguistic prestige; Linguistic stigmatization.
\end{abstract}

Recibido: 15-09-2020

Aceptado: 09-11-2020

\footnotetext{
${ }^{1}$ Este trabajo se enmarca en el proyecto de investigación "La atenuación lingüística en el español chileno: enfoque pragmalingüístico y sociopragmático" (ANID/CONICYT Fondecyt Regular 1200003).
} 


\section{Introducción}

Desde mediados del siglo XX surgió una serie de propuestas que conciben al lenguaje como un recurso de la cultura, como un elemento que se construye en el seno de la actividad social y que no se reduce a un acto de comunicación, siendo en sí mismo una práctica cultural (Duranti). La consideración del lenguaje como un fenómeno de naturaleza sociohistórica y performativa implicó buscar el sentido y significado último de la comunicación en el complejo entramado que une al lenguaje y la sociedad.

En este sentido, para comprender la actividad lingüística y comunicativa es ineludible la consideración de todos los elementos que la rodean y componen. Labov sostuvo, por ejemplo, que el cambio lingüístico tiene una base social como fundamento y que, por lo mismo, responde a dimensiones subjetivas, a las características particulares de los hablantes y a fenómenos de naturaleza global.

En la tradición investigativa, específicamente del ámbito fonético-fonológico, uno de los conceptos clave para estudiar sus variaciones ha sido el prestigio lingüístico. Distintos autores (Labov; Silva y Enrique; Moreno Fernández), tomando los aportes ofrecidos por la sociología y por el desarrollo de las teorías de la estratificación social, han explicado la propensión y valoración subjetiva que los hablantes muestran hacia una u otra forma de hablar a partir del prestigio asociado a las variantes tanto fonéticas, léxicas, sintácticas y/o discursivas. Este concepto, tratado por la sociología estructuralista funcional, ha contribuido en los estudios del lenguaje en tanto establece una categoría teórico-analítica para el abordaje de estos fenómenos de estudio.

En el caso de Chile, los estudios sociofonéticos iniciaron en la década del setenta un recorrido que continúa hasta la actualidad (Ortiz y Saavedra). Recientemente, Figueroa y Sadowsky han centrado sus estudios en la variación sociolectal de los fonemas consonánticos del español de Chile, constatando, entre otras cosas, que el estado de las variantes, que algunos circunscriben al ámbito de la lingüística pura, se relaciona directamente con las valoraciones subjetivas y sociales. Respecto al grupo fonemático /tr/ y los fonemas $/ \overline{\mathrm{r}} /, / \mathrm{g} / \mathrm{y} / \mathrm{j} /$, han descrito e inventariado sus variantes alofónicas, así como también, las han situado en una escala de prestigio y estigmatización en relación, principalmente, con las variables sociales de estrato socioeconómico, género y grupo etario. 
Sadowsky afirma que existiría una nivelación sociolectal de los alófonos estudiados, influenciada posiblemente por razones estilísticas y sociales. Complementariamente, Figueroa plantea la existencia de un eje oclusión-prestigio/fricción-estigma que influiría en el uso que los hablantes realizan sobre variantes de estos tipos.

Estos estudios han logrado generar un mapa descriptivo con relación a las particularidades en las cuales opera el prestigio lingüístico. Un desafío relacionado a lo que se ha indagado en la materia sería examinar las valoraciones sociales de las variantes alofónicas considerando sus contextos fonéticos inmediatos, buscando comprender las implicancias contextuales en el uso y estado social y subjetivo de las mismas.

\section{Marco conceptual}

\subsection{Prestigio lingüístico}

El concepto de prestigio ocupa un lugar importante en la comprensión de la actividad lingüística. En esta línea, si hablamos de prestigio lingüístico, estamos refiriéndonos, en esencia, a motivos sociales (Labov; Alvar; Salazar; Falcón y Mamani). Bajo esta premisa, se deduce que el proceso valorativo de los hablantes hacia su dialecto o forma de hablar constituye un proceso complejo y dialéctico entre los sujetos y sus motivaciones, y entre estos y los procesos sociales en los cuales están inmersos (López; Castillo).

A partir de lo anterior, el prestigio, en tanto conjunto sistemático de creencias y valoraciones subjetivas y sociohistóricas, y como categoría de análisis, consistiría en un razonamiento dinámico, mudable, situado y voluble, siendo otorgado sin responder necesariamente a la propia virtud del objeto en cuestión, sino que, por el contrario, su justificación podría estar en el momento y situación misma de la evaluación, valiéndose de cuestiones de índole política y/o moral (Hernández Campoy).

El prestigio lingüístico podría explicar la actividad lingüística de los hablantes y, con ello, establecer su operatividad, la que estaría relacionada con “obtener unos legítimos derechos a ser respetados; pasar luego al reconocimiento general e imponer, más tarde, aquello que se ha conseguido" (Alvar 15).

El proceso operativo del prestigio continuaría, atendiendo a su carácter dinámico e inestable, a la manera del eterno retorno planteado por Nietzsche. De esta manera, el transcurrir repetitivo de la vida y, para estos efectos, de la actividad lingüística, daría paso a 
Artículo. Andrea Carrasco. Variación sociofonética del español de Chile: percepciones subjetivas de futuros profesores del sistema

un proceso en el que la revolución y cambio lingüístico vuelven a comenzar (López; Salazar).

Por las razones mencionadas, los valores atribuidos a una u otra forma de hablar no serían puramente lingüísticos, pues en ellos se integran la autoridad literaria, situaciones de instrucción, la corrección aceptada, motivos históricos, etcétera. El prestigio de una lengua, por lo tanto, está cruzado por una infinidad de razones actuantes, extralinguiísticas, motivacionales y socioculturales que determinan su prevalencia o sustitución (Salazar; Silva y Enrique).

\subsection{Actitudes lingüísticas}

Concebir la lengua como un hecho de carácter social implica entender que el devenir de la misma está estrechamente ligado a sus usuarios y a los sistemas culturales y simbólicos en los que estos se encuentran. De esta manera, los hablantes, a la vez que participan de creencias establecidas y aceptadas socialmente, reaccionan ante estas constituyendo un potencial de transformación individual (Serrano).

Cuando se ha estudiado la lengua con una perspectiva histórica o comparativa, uno de los factores que ha tenido incidencia en los procesos de variación o cambio está relacionado con las reacciones o actitudes subjetivas que los hablantes adoptan hacia una u otra forma lingüística. Sin embargo, la naturaleza de las actitudes subjetivas ha supuesto un reto para los investigadores de corriente positivista, debido a la dificultad de medición y a que exige la aceptación de que esta se puede visualizar mediante una determinada conducta (Serrano). Por otra parte, en la investigación de enfoque mentalista se superan las dificultades mencionadas, puesto que se conciben las actitudes como un elemento intermedio e interno de los individuos, que media entre el estímulo presentado y sus respuestas (Blas).

El concepto de actitud lingüística surge del campo de la psicología social, dentro del cual se entiende como "la disposición para reaccionar favorable o desfavorablemente hacia una clase de objetos" (Edwards 20). En este campo, se establece que toda actitud lingüística incluye o comprende una serie de creencias e ideas que le dan sustento y coherencia.

En el campo de la sociolingüística, el estudio de las actitudes lingüísticas busca entender o explicar cómo el factor sociopsicológico del hablante incide en el uso de la lengua y sus variantes. En este sentido, los estudios sociolingüísticos que recurren a las actitudes lingüísticas lo han hecho tradicionalmente para conocer y describir el estatus de las 
variantes que presenta el sistema o para correlacionarlas con los grupos sociodemográficos que las utilizan. Al mismo tiempo, las actitudes que los hablantes adquieran hacia determinadas formas nos permitirán comprender el proceso de cambio lingüístico, conservación, ultracorrección, y adquisición de segundas lenguas (Falcón y Mamani, Hernández y Dinamarca).

\section{Metodología}

\subsection{Sujetos de la muestra}

El universo de esta investigación se conforma por estudiantes de pedagogías de una universidad estatal de la región Metropolitana. En este punto, es importante mencionar que el estudio de las valoraciones de profesores constituye un saber que posibilitaría la comprensión de su proceder pedagógico (Ramírez).

La muestra se constituyó salvaguardando el rigor y validez de las percepciones recogidas, por lo que se estableció como criterio de exclusión la formación disciplinar en lingüística, específicamente, en fonética. Este criterio se justifica en la naturaleza de nuestro objeto de estudio y en la metodología escogida, ya que, para lograr acceder a las valoraciones subjetivas de los informantes, se optó por una aproximación de carácter indirecto. El hecho de acceder al nivel subconsciente de los informantes posibilitaría acceder a evaluaciones menos contaminadas por ideas preconcebidas o por lo que el informante cree que se espera que responda. Debido a lo anterior, la población del presente estudio excluye a los estudiantes pedagogías de castellano, francés, inglés, alemán y educación diferencial, mención trastornos de la audición. Considerando los criterios de inclusión/exclusión presentados, la presente investigación contó con la participación de 120 sujetos.

\subsection{Instrumento de recolección de datos y aplicación}

La técnica de aproximación y de recolección de datos fue la técnica de pares falsos (matched-guise technique). En torno a esta, Hernández y Almeida afirman que su utilización tradicional ha sido para comprender y analizar las actitudes lingüísticas y las valoraciones subjetivas que asignan los hablantes a ciertas variedades y variantes lingüísticas presentadas. 
El instrumento utilizado requirió de la construcción de estímulos sonoros que incluyeran las variantes de cada fonema seleccionando, los cuales presentan variaciones en sus elementos constitutivos, en el grado de fricación y en la sonoridad. Estas variantes fueron evaluadas en contextos intervocálico, inicial absoluto, prepausal, preconsonántico y postconsonántico. Los estímulos se tomaron de un corpus confeccionado por los autores, en el cual se incluye el fenómeno a estudiar, insertando uno por cada frase u oración, y sin que se entrecrucen en un mismo estímulo.

En la recolección de datos se utilizó el Test de Evaluación Subjetiva construido por Valdivieso, el cual, a su vez, está inspirado en el modelo de recolección de datos de Labov para su estudio del inglés de Nueva York.

A continuación, a modo de evidencia, se observan algunas de las realizaciones estudiadas en contexto intervocálico, correspondientes a los estímulos "Mi primo cumplió treinta años", “Me llamaron del hospital”, "Nunca prendo la radio” y “¿Cuánta leche queda?”.
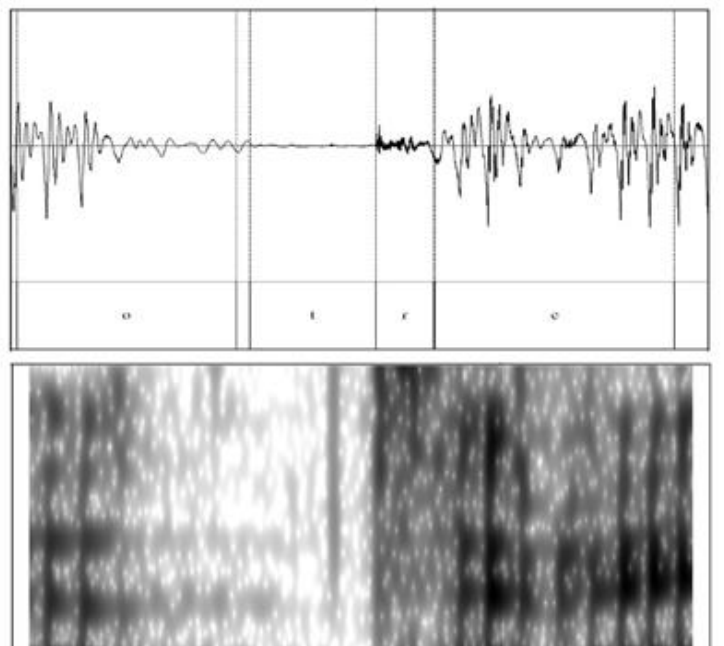

Ilustración 1: Oscilograma y espectrograma de [tr] en contexto intervocálico
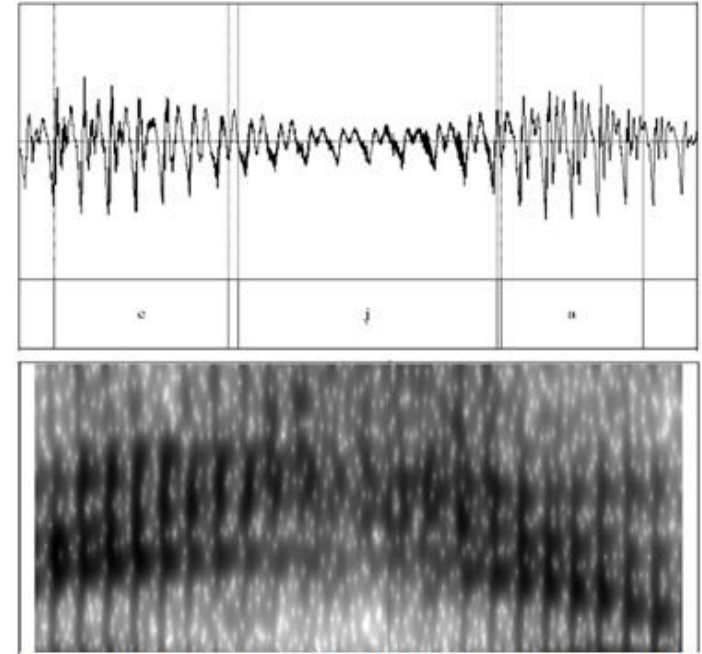

Ilustración 2: Oscilograma y espectrograma de [eja] en contexto intervocálico 

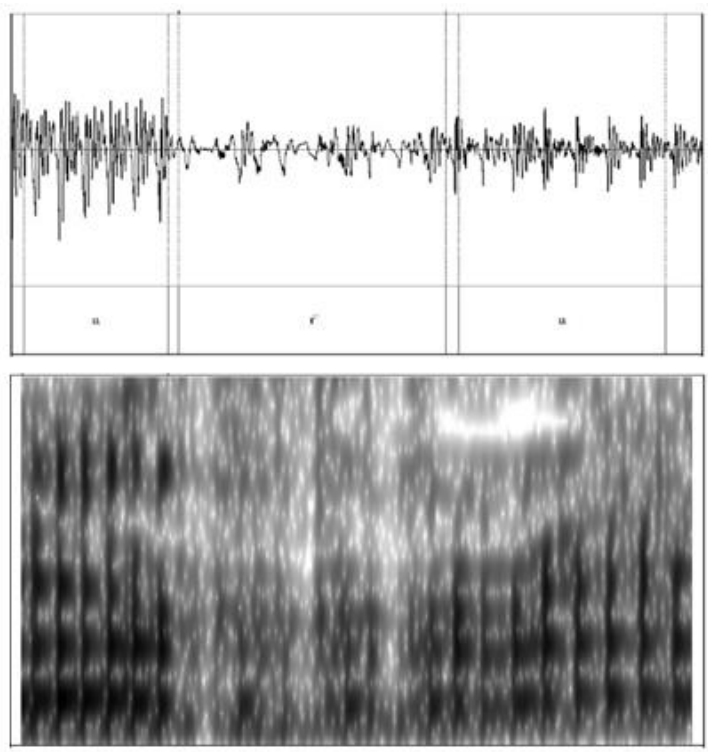

Ilustración 3: Oscilograma y espectrograma de [ara] en contexto intervocálico
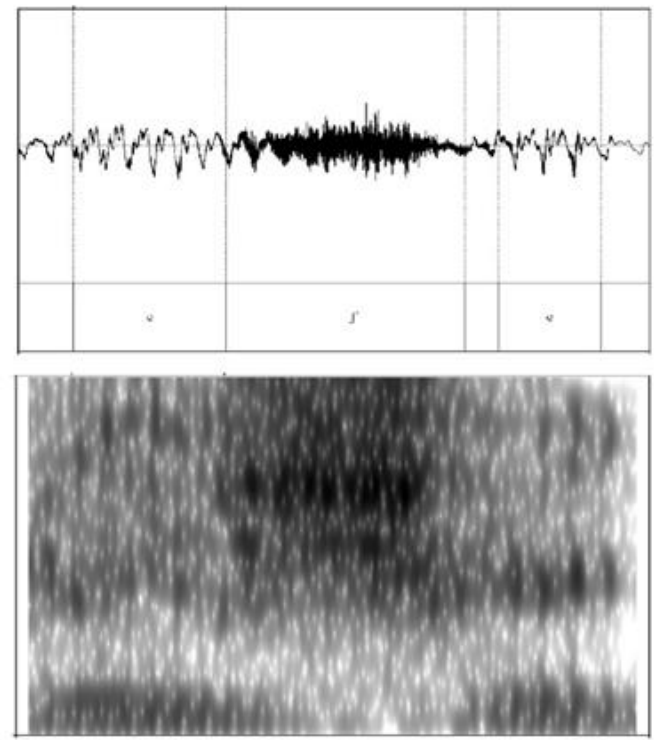

Ilustración 4: Oscilograma y espectrograma de [J] en contexto intervocálico

\subsection{Análisis}

Los datos recolectados se organizaron de manera factorial, atendiendo a las valoraciones otorgadas en cada estímulo. Posteriormente, se exportó esta información al programa estadístico de análisis de datos SPSS (Statistical Package for the Social Sciences), con el cual se generaron tablas y gráficos de carácter descriptivo.

\section{Presentación y discusión de resultados}

En este apartado, se entregarán los resultados obtenidos organizados por fonema o grupo fonemático. Los valores entregados por los informantes oscilan entre los valores 1,0 y 6,0. Se establecieron como evaluaciones no marcadas sociolingüísticamente aquellas que se ubican en el rango [3,6-4,5].

\subsection{Valoraciones subjetivas asociadas a realizaciones de / $\mathrm{tr} /$}

Los estímulos presentados a los informantes consideran distintos contextos en español, los que en el caso del grupo /tr/ son intervocálico, inicial y postconsonántico (ver gráfico 1). Considerando los contornos posibles y las variantes estudiadas, el total de estímulos valorados fueron nueve. 
Según las percepciones recogidas, se observa una curva descendente conforme a) se reemplaza la realización vibrante simple por la fricación y b) conforme se pierde la sonoridad. Por esta razón, las valoraciones más altas son otorgadas a la realización canónica $[4,6]$, alcanzando el estatus de prestigio, aunque ceñidamente y solo en contexto postconsonántico.

Como se mencionó, la realización estándar [tr] del grupo /tr/ no levanta juicios ampliamente prestigiosos, su valoración en los demás contextos evaluados, inicial e intervocálico, no es marcada, siendo, por lo demás, estadísticamente irrelevante la diferenciación entre estos dos contornos, en tanto alcanzan valores adyacentes: [3,9] y [3,8] respectivamente.

El alófono láminoalveolar africado áfono [t. muy acotado de la banda, el cual marca su extremo inferior en el punto [3,4] y su extremo superior en el $[3,6]$. Las estrechas dos décimas en las que se distribuyen las valoraciones de este alófono reflejan un estatus no polarizado, a pesar de estar en la categoría de estigma en los contextos inicial y postconsonántico. Sin embargo, la proximidad entre estos y el estatus no marcado pondrían en tensión las afirmaciones que aseguran que este alófono es ampliamente rechazado (Lenz; Sadowsky, El inventario). Al mismo tiempo, la colindancia de los contornos estudiados revelaría, por lo menos para esta realización, ser insustancial a juicio de los informantes.

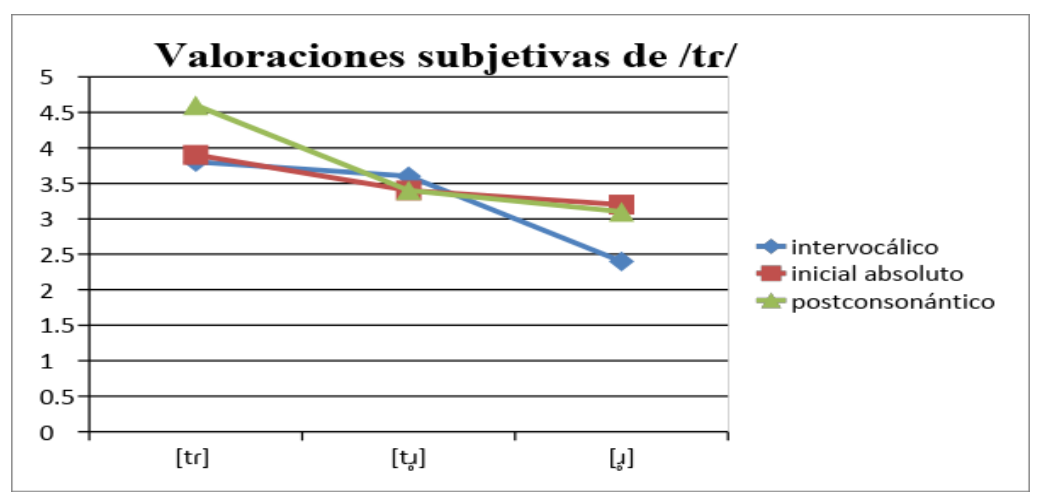

Gráfico 1. Valoraciones subjetivas asociadas a realizaciones de /tr/

Respecto al último fono estudiado, el cual pierde completamente el elemento oclusivo y la sonoridad del elemento fricativo [II.], se observa un rechazo generalizado en todos los 
contornos fonéticos estudiados. Sin embargo, en contexto inicial se da la realización más elocuentemente despreciada por los hablantes, marcando el punto más bajo de las valoraciones para este grupo: [2,4]. Un hallazgo interesante es el grado de estigmatización otorgada en los demás contextos, puesto que, si bien se encuentran en el estatus de estigma sociolingüístico, estos no fueron categóricos, divergiendo significativamente con quienes lo describen como asociado al estrato social muy bajo (Figueroa, Soto-Barba y Nanculeo; Sadowsky).

Por último, se observan algunos comportamientos interesantes de puntualizar, como la concurrencia de las valoraciones de [t.I] en todos sus contextos, los cuales se sitúan en torno al $[3,4]$; la gran proximidad de los valores para [II ] en los contextos inicial y postconsonántico, y el distanciamiento considerable del contexto postconsonántico en la realización de $[\mathrm{t} c]$, constituyendo, por lo demás, el alófono y contorno mayormente aceptado por los informantes.

\subsection{Valoraciones subjetivas asociadas a realizaciones de /j/}

El estudio del fonema prepalatal fricativo sonoro /j/ consideró los contextos intervocálico, inicial y postconsonántico. Teniendo en cuenta estos contornos y las variantes estudiadas, el total de estímulos valorados fueron nueve.

Las valoraciones recogidas se distribuyen en un rango acotado del gráfico (ver gráfico 2), oscilando entre los puntos $[2,4]$ y $[4,6]$. En relación con estos resultados, y en concordancia con estudios previos, ocho de los nueve estímulos presentados fueron considerados no marcados. De esta tendencia se desprende un estatus no estereotipado ni significativo en términos sociolinguiísticos. La mayoría de las realizaciones, por lo tanto, no gesta ni alberga juicios para sectores amplios de la sociedad.

A pesar del estrecho rango en el que se movieron las valoraciones recogidas, es posible distinguir algunas inclinaciones interesantes de analizar. Una de ellas es la categoría alcanzada por el noveno estímulo presentado, correspondiente a la realización fricativa lenis en contexto intervocálico. Esta realización se consideró prestigiosa [4,8] por los informantes, estableciéndose una disparidad con los hallazgos de Sadowsky y Salamanca, quienes la proponen como no marcada. 
Por otra parte, se observa un patrón estable entre la realización fricativa fortis y la fricativa lenis, dentro del cual, la realización lenis fue siempre mejor evaluada. En la misma línea, cabe puntualizar que las curvas de ascendencia no son gradualmente idénticas. Se distingue un ángulo mayor en el contexto intervocálico, le sigue el postconsonántico y, finalmente, el inicial. Estas diferencias pueden atribuirse al contorno fonético, puesto que, relacionando los mismos factores, en este caso, las realizaciones fortis y lenis, los grados de aceptación o rechazo varían en tanto varía el contexto en el que se insertan.

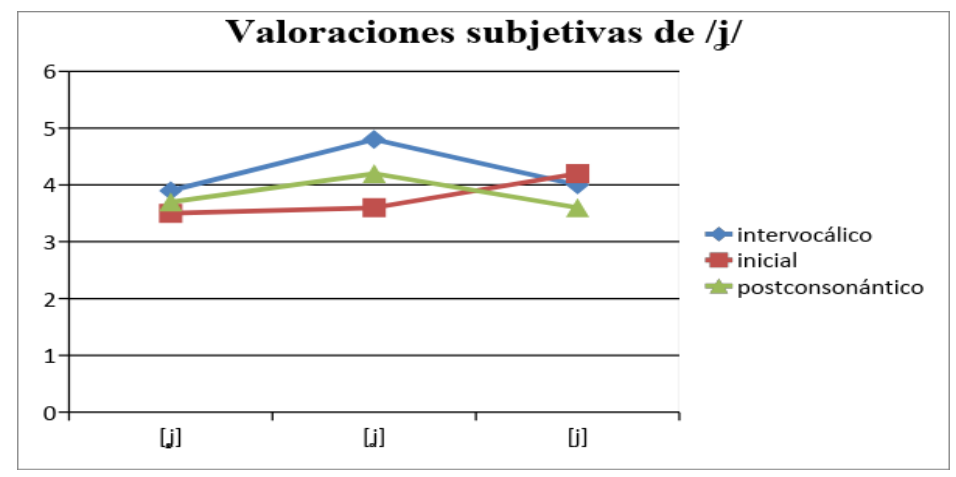

Gráfico 2. Valoraciones subjetivas asociadas a realizaciones de /j/

La relación entre la realización fricativa lenis y la aproximante conforma un patrón descendiente solo en los contextos intervocálico y postconsonántico. En el contexto inicial, se forma una curva inversa, en donde la realización aproximante es mejor valorada que la fricativa lenis.

Entre la realización fricativa fortis y la aproximante se observan valoraciones muy similares, prácticamente idénticas, y al igual que en la relación anterior, solo en los contextos intervocálico y postconsonántico. La relación de la fricativa con la aproximante vuelve a generar una curva divergente, moviéndose ascendentemente conforme disminuye la fricción articulatoria.

\subsection{Valoraciones subjetivas asociadas a realizaciones de $/ \overline{\mathrm{r}} /$}

La investigación en torno al fonema láminoalveolar vibrante múltiple sonoro se realizó considerando los contextos intervocálico, inicial, postconsonántico y final. Dadas las 
particularidades que la actividad lingüística presenta en el contexto final, este se analizó separadamente con la finalidad de ilustrar con mayor claridad las diferencias sociofonéticas de una u otra variante (ver gráfico 4). Por lo tanto, teniendo en cuenta los contornos y variantes estudiadas, el total de estímulos valorados fueron trece.

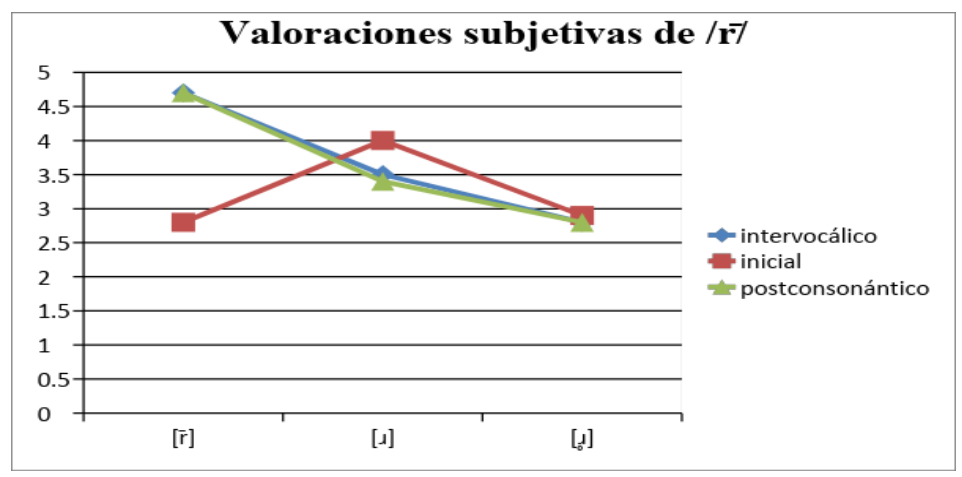

Gráfico 3. Valoraciones subjetivas asociadas a realizaciones de $/ \overline{\mathrm{r}} /$

Las valoraciones recogidas que muestra el gráfico 3 se distribuyen mayoritariamente en las categorías no marcada y de estigma sociolingüístico, lo que coincide, en primera instancia, con los hallazgos de la literatura especializada. Sin embargo, en este estudio, se establece una discrepancia en torno a las valoraciones de $[\overline{\mathrm{r}}]$ en todos los contextos analizados. Según estudios realizados por Bobadilla y Bobadilla y Bernales, el alófono canónico se consideraría no marcado. A pesar de ello, los resultados de este estudio lo categorizan como prestigioso en contexto intervocálico y postconsonántico, y estigmatizado en contexto inicial.

Por otra parte, se distingue un continuo decreciente entre las realizaciones vibrante múltiple sonora $[\overline{\mathrm{r}}]$, fricativa sonora $[\mathrm{I}]$ y fricativa áfona $[\mathrm{I}]$, aunque solo en los contextos intervocálico y postconsonántico. Como se observa, estos contornos vuelven a generar valoraciones coincidentes e idénticas. Al mismo tiempo, las realizaciones de $/ \overline{\mathrm{r}} /$ en contexto inicial son nuevamente disidentes respecto a los otros dos contextos.

En relación con el alófono láminoalveolar fricativo áfono [⿳્口䒑彡], tradicional y transversalmente estigmatizado, este estudio no muestra hallazgos nuevos. El alófono en cuestión fue 
rechazado en todos los contextos estudiados, por lo que se confirma el estigma reportado en estudios previos.

Como se anticipó al principio, el contexto final fue analizado de manera separada, en atención a la alternancia entre articulación vibrante múltiple/fricativa y sonora/áfona.

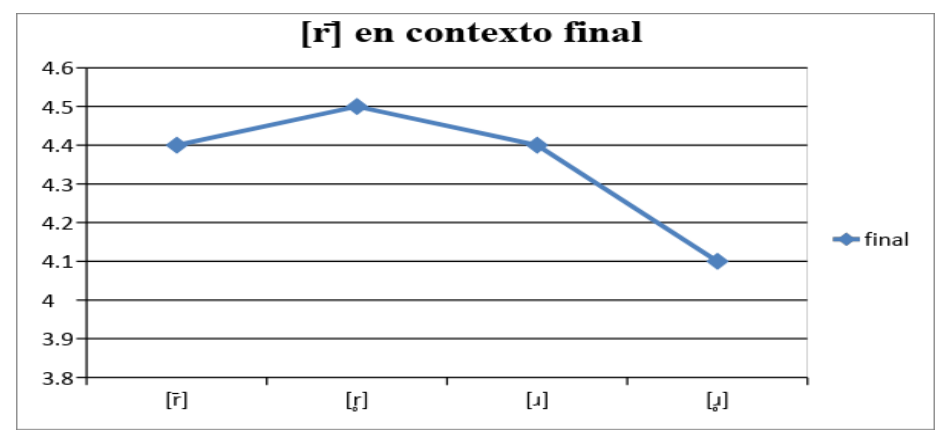

Gráfico 4. Valoraciones subjetivas asociadas a realizaciones de $/ \overline{\mathrm{r}} /$ en contexto final

El análisis apartado de la vibrante múltiple en contexto final permite identificar algunas tendencias relevantes. En primer lugar, se registra una ambivalencia en la valoración de la pérdida de sonoridad, puesto que, en el caso de las vibrantes, el paso de la realización sonora a la áfona genera una subida en las valoraciones, mientras que, en el caso de las fricativas, se genera una disminución pronunciada de las valoraciones asignadas en este contexto.

Por otra parte, el comportamiento de las valoraciones otorgadas a las realizaciones sonoras también es llamativo. Como se observa en el gráfico 4, se establece una relación de igualdad entre las valoraciones de la vibrante sonora y la fricativa sonora. Al parecer, los informantes no distinguen el cambio articulatorio que afecta al fono en cuestión, o también, no les es significativo, puesto que evalúan ambas realizaciones en el punto [4,4], posicionándolas como realizaciones no marcadas sociolingüísticamente.

4.4.Valoraciones subjetivas asociadas a realizaciones de $/ \mathrm{t} /$

Para estudiar las valoraciones asociadas a las realizaciones del fonema postalveolar africado áfono [j], se consideraron los contextos intervocálico, inicial, postconsonántico, final y preconsonántico. Al correlacionar los cinco contextos mencionados con las tres variantes de $/ \mathfrak{g} /$, se alcanza un total de 15 estímulos analizados. 
Al observarse el gráfico de valoraciones número 5, lo primero que llama la atención es el rango en el que se distribuyen los valores recogidos. Si se tienen en cuenta los estereotipos y prejuicios que se han reportado para algunas realizaciones de / $\mathfrak{g} /$, sería admisible una distribución más extrema o polarizada. En este estudio, por el contrario, la amplitud que se alcanza podría asociarse a fonemas del español menos estereotipados sociolingüísticamente, como lo son las distribuciones de /d/ o /j/. Si comparamos la extensión de la banda de /j/ en este estudio, la cual abarca los puntos [2,4] y [4,6], con la extensión que alcanza / $/ \mathrm{g} /$, la cual abarca los puntos [2,8] y [4,7], se puede percibir la gran similitud entre ambos conjuntos. Tal como se mencionó previamente, esta similitud sorprende y tensiona los hallazgos que han reportado Bobadilla y Bobadilla, Valdivieso ( $E l$ español), Cepeda o Sadowsky y Salamanca. Estos autores mencionan que las realizaciones de / $\mathrm{g} /$ suscitarían valoraciones y representaciones sociales marcadas y hasta estereotipadas, asociándolas con los estratos altos, bajos y muy bajos, percepciones que no se ven reflejadas en las tendencias obtenidas en este trabajo.

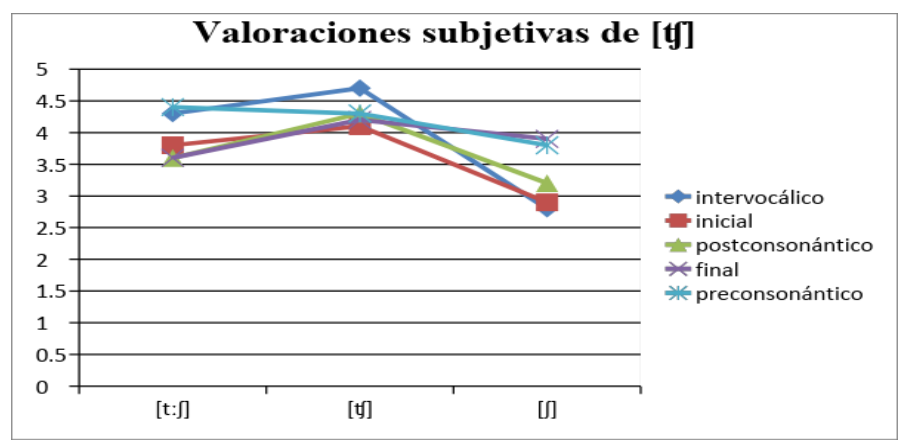

Gráfico 5. Valoraciones subjetivas asociadas a realizaciones de $/ \mathfrak{t} /$

Respecto a los mismos estudios mencionados, en los cuales se ha encontrado un mayor grado de aceptabilidad y prestigio en las realizaciones con prominencia oclusiva, este estudio difiere significativamente. La realización [t: $\left.\int\right]$, con prominencia oclusiva, no fue considerada prestigiosa en ninguno de los contextos estudiados. Los valores alcanzados corresponden en su totalidad a estimaciones no marcadas.

Por otra parte, si se relacionan los valores de [t: $\left.\int\right]$ con los de [t] , considerada canónica y no marcada según la bibliografía citada, la curva que se observa es inversa a la reportada en otros estudios y otras zonas geográficas. Concretamente, en los contextos intervocálico, 
inicial, postconsonántico y final los valores de la realización canónica son mejores evaluados y mayormente aceptados que la realización con prominencia oclusiva. Solo en el contexto preconsonántico se establece una curva acorde a los estudios mencionados y al eje de oclusión-prestigio/fricción-estigma propuesto por Figueroa.

Los resultados en torno al alófono postalveolar fricativo áfono [S], variación que pierde completamente el elemento oclusivo, son verdaderamente inesperados. Si bien los contextos intervocálico, inicial y postconsonántico fueron rechazados por los informantes, hubo dos contextos en los que no se reconoce el fuerte estigma que se le atribuye, así como tampoco su constitución como "el estereotipo lingüístico más emblemático del castellano chileno” (Sadowsky 74). Los contextos en los que el alófono [S] no fue seguido de un sonido vocálico se evaluaron como no marcados.

Finalmente, incluso en los contextos en que la realización [ $\left.\int\right]$ no se estigmatiza, la pérdida del elemento oclusivo se considera un factor de descenso en las percepciones recogidas. Solo en el contexto final la curvatura es inversa, puesto que la realización con prominencia oclusiva es peor valorada que la fricativa.

\section{Discusión}

A modo general, algunos de los resultados obtenidos concuerdan con las tendencias reportadas previamente, en las que los hablantes rechazan y estigmatizan fonos áfonos fricativizados. Sin embargo, entre los resultados obtenidos también se advierten desplazamientos estimativos que no coinciden con los estudios sociofonéticos anteriores, dado que algunas de las curvas de aceptación o rechazo obtenidas son, incluso, inversas a las reportadas en anteriores investigaciones. Asimismo, se identifican algunos contextos lingüísticos en los que se reiteran significativamente actitudes de rechazo o aprobación, constituyéndose como contextos fonéticos socialmente marcados. Para el caso del rechazo o estigma sociolinguiístico, se identifican el inicial absoluto y el intervocálico, y en el caso del prestigio, se registra el final o prepausal.

A continuación, en relación con los principales hallazgos de esta investigación, se establece un diálogo con el trabajo realizado por Figueroa, en el cual se establece la existencia de un eje en el sistema sociofónico del español de Chile, el que correlaciona las articulaciones oclusiva y fricativa con valoraciones de prestigio y estigma, respectivamente. En este trabajo, contrario a los planteamientos del eje, las relaciones que se establecen entre las 
variantes presentadas y las percepciones en torno a ellas no son sistemáticas ni podrían amparar lecturas generales. Particularmente, los puntos de desencuentro con los

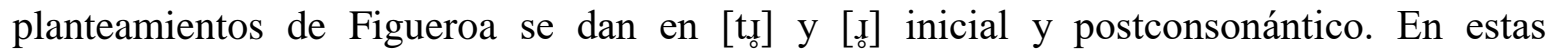
realizaciones, los informantes evaluaron idénticamente los dos alófonos, a pesar de que se elimina el elemento oclusivo en el segundo, lo que constituye una distancia significativa con los planteamientos del eje oclusión/fricción antes mencionado. En las realizaciones de /j/, tampoco se cumplen los postulados del eje, ya que en contexto postconsonántico las valoraciones fueron iguales para [j], [j] y [j], sin importar la gradación fricativa que los distingue. Lo mismo ocurre en el caso de $/ \mathfrak{g} /$, puesto que se establece un enroque en las valoraciones de $\left[\mathrm{t}: \int\right]$ y $[\mathfrak{t}]$ en contexto postconsonántico. Por último, en contexto final, las evaluaciones de $[\mathfrak{f}]$ y $\left[\int\right]$ resultaron ser idénticas, a pesar de que se elimina la oclusión en una de las realizaciones.

Por otra parte, la descripción sociofonética de las consonantes del español de Chile realizada por Sadowsky ofrece un estudio detallado de las variantes que aquí también se refieren. Respecto al grupo consonántico /tr/, el autor afirma que la realización canónica de este fonema es valorada según el estrato socioeconómico del informante. En este sentido, [tr] se consideraría generalmente como prestigioso, mientras que los informantes de estrato socioeconómico alto, la estigmatizarían. En esta investigación, las valoraciones se analizaron de acuerdo con el modo articulatorio y al contexto fonético en el que se insertaron los sonidos. Según esto, el alófono en cuestión obtuvo prestigio en contexto postconsonántico y estigmatización en contexto intervocálico.

Las valoraciones de / $\mathrm{j} /$, por otro lado, son escasamente coincidentes con los planteamientos de Sadowsky, quien plantea que [j] es considerado significativamente estigmatizado, ante lo cual esta investigación aporta un matiz interesante de mencionar: el alófono [j] fue estigmatizado solo en contexto inicial absoluto, en todos los demás contextos fue valorado como realización no marcada.

Respecto a las realizaciones de $/ \overline{\mathrm{r}} /$, se observan diferencias entre los planteamientos de Sadowsky y los resultados de este estudio. Según el autor, las puntuaciones de las realizaciones oscilarían entre las valoraciones de no marca y estigma sociolingüístico. Sin embargo, en este trabajo se encontraron valoraciones que consideran prestigiosas algunas realizaciones de $[\overline{\mathrm{r}}]$ intervocálica, postconsonántica y final, y de $[\overline{\mathrm{r}}]$ en contexto final. 
Finalmente, con respecto a las valoraciones de /ty/, la presente investigación coincide parcialmente con los planteamientos de Sadowsky, posiblemente por la consideración del contorno fonético en el análisis de las valoraciones. En el caso de [t]], el autor afirma que es una realización no marcada sociolingüísticamente, sin embargo, en este trabajo, lo anterior se cumple solo en contexto inicial y final. El alófono [t:f], considerado generalmente como una realización emblemática del estrato alto (Sadowsky) y, por lo tanto, prestigiosa, entregó datos interesantes: solo en contexto intervocálico y preconsonántico alcanzó cifras de prestigio, en sus demás contextos obtuvo una evaluación no marcada. Por último, el alófono [S], descrito por Sadowsky como una realización fuertemente estigmatizada, también arrojó resultados inesperados, ya que fue aceptado y no marcado en sus contextos postconsonántico, final y preconsonántico.

\section{Conclusiones}

Se advierte que el contorno linguiístico es relevante y significativo en la conformación de juicios y tendencias sociolinguísticas. Al mismo tiempo, los resultados obtenidos se relacionan coherentemente con la Teoría de las Connotaciones Sociales (Giles), según la cual los fenómenos lingüísticos no portarían en sí mismos valores sociolingüísticos inherentes, sino que estos serían otorgados culturalmente y en situaciones concretas de uso. El criterio de sonoridad también fue relevante en las valoraciones entregadas por los informantes, quienes, al ser enfrentados a realizaciones sonoras y luego a sus pares áfonas, actuaron desaprobatoriamente, llegando, incluso, a estigmatizar el segundo fono presentado.

Por otra parte, las valoraciones prestigiosas no muestran en todos los casos concordancia con el marco de investigaciones referido. En esta oportunidad, el estatus de prestigio fue otorgado de manera discontinua, por lo que proponer patrones podría obedecer a interpretaciones apriorísticas o casuales. Concretamente, se consideraron prestigiosas las

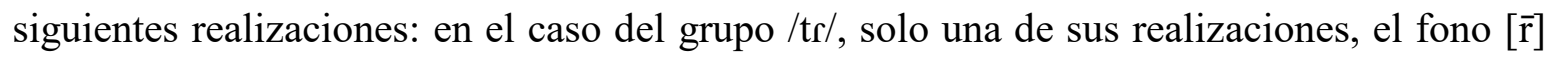
en posición postconsonántica; en el caso del fonema $/ \overline{\mathrm{r}} /$, el canónico $[\overline{\mathrm{r}}]$ en los contextos intervocálico, postconsonántico y final; en el caso de $/ \mathfrak{g} /$, dos de los tres alófonos presentados, [t:f] en posición intervocálica y preconsonántica, $\mathrm{y}[\mathrm{g}]$ en contexto intervocálico, postconsonántico y preconsonántico, por último, en el fonema $/ \mathrm{j} /$, las realizaciones [j] intervocálica y [j] inicial absoluta. 
Algo similar ocurre con las asignaciones de estigma lingüístico. Como se mencionó en la presentación de resultados, algunas realizaciones que han sido ampliamente rechazadas en otros estudios, en este fueron consideradas no marcadas o levemente estigmatizadas, aunque, cabe precisar que estos resultados se dieron solo en algunos contornos, por lo que no podrían formularse deducciones categóricas a partir de ellos. A pesar de lo anterior, sí son admisibles lecturas parciales, como que algunos contextos encubrirían el rechazo generalizado de realizaciones tradicionalmente estigmatizadas. De esta manera, las realizaciones estigmatizadas son las siguientes: en el grupo consonántico /tr/, los alófonos [t.I] y [II] en todos sus contextos, mientras que el canónico [tr] solo en contexto

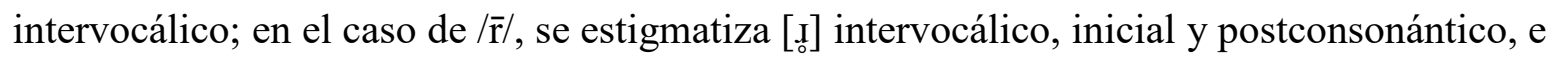
inesperadamente $[\overline{\mathrm{r}}]$ en contexto inicial, aunque no de manera contundente; de las realizaciones de / $\mathrm{y} /$, la versión fricativa [S] en los contornos intervocálico e inicial absoluto, $y$, finalmente, de los alófonos presentados de /j/, solo la variante [j] en su contexto inicial, aunque no en un porcentaje mayoritario.

Lo mencionado hasta este punto nos advierte de algunas discrepancias con reportes y estudios previos. Las diferencias metodológicas podrían ciertamente justificar la emergencia de las nuevas tendencias sociolingüísticas descritas. Lo mismo podrían las diferencias en el análisis e interpretación de datos. Sin embargo, creemos que el uso de aproximaciones metodológicas y analíticas variadas también podría enriquecer la comprensión de estos fenómenos, en tanto posibilitan una mirada caleidoscópica del objeto de estudio, por lo que atribuir las variaciones entre unas y otras investigaciones a la elección metodológica sería caer, de cierto modo, en un atrincheramiento investigativo. En coherencia con lo anterior, creemos que un elemento clave para comprender los matices y variaciones reportadas en este trabajo es la consideración de los informantes/hablantes como actores sociales, lo que, a su vez, exige pensar la actividad lingüística como una actividad eminentemente social (Duranti). En este punto, cabe precisar que cualquier actividad social, y linguiística, se enmarca en una comunidad sociocultural específica, y que esta condiciona y reproduce las relaciones simbólicas que en ella operan (Hymes).

Por último, conviene interpretar las particularidades que muestran los hallazgos de esta investigación como una posible huella de las particularidades ideológicas y políticas que pudiese representar el conglomerado de profesores en el contexto chileno actual, 
entendiendo que este históricamente ha participado en mayor o menor medida en las coyunturas políticas y sociales devenidas (Reyes), su importancia como sujeto de estudio, por lo tanto, es singular dentro del espectro de profesionales de otras áreas. En este sentido, ni los hablantes ni las palabras son elementos socialmente neutros, puesto que su naturaleza los enlaza con el contexto sociocultural en el que se enuncian, desplegándose en este acto el significado indicial y, por lo tanto, sociocultural de los signos lingüísticos.

\section{Referencias bibliográficas}

Alvar, Manuel. "La lengua, los dialectos y la cuestión del prestigio". Estudios sobre variación lingüística. Ed. Francisco Moreno. Salamanca: Universidad de Alcalá de Henares, 1990: 13-26.

Bernales, Mario. "La actividad científica de Rodolfo Lenz en Chile", Cuadernos de Lengua y Literatura (Homenaje al Dr. Rodolfo Lenz) 1 (1988): 9-19.

Blas Arroyo, José Luís. Sociolingüística del español: desarrollos y perspectivas en el estudio de la lengua española en contexto social. Madrid: Cátedra, 2005.

Bobadilla, Félix y Bobadilla, Gustavo. "El comportamiento de las variables lingüísticas /tr/, $/ \overline{\mathrm{r}} / \mathrm{y} / \mathrm{ts} /$ en el área urbana de Rancagua", Cuadernos de lenguas modernas 2 (1979): 18-24.

Castillo, Mario. El estudio de las actitudes lingüísticas en el contexto sociocultural: el caso del mexicano de Cuetzalan, Anales de Antropología 40.1 (2006): 283-317.

Cepeda, Gladys. Las consonantes de Valdivia. Valdivia: Universidad Austral de Chile, 1991.

Duranti, Alessandro. Antropología Lingüística. Madrid: Cambridge University Press, 2001.

Edwards, J. Language attitudes and their implications among English speakers. Ed. H. R. Bouchard y H. Giles. Londres: Arnold, 1982.

Falcón, Pedro y Mamani, Luis. Actitudes lingüísticas en contextos interculturales: población Asháninka Bajo Chirani. Revista de linguiística teórica y aplicada 55.1 (2017): 95-115.

Figueroa, Mauricio, Soto-Barba, Jaime. y Nanculeo, Marco. Los alófonos del grupo consonántico /tr/ en el castellano de Chile. Onomázein 22 (2010): 11-42.

Figueroa, Mauricio. El Eje Oclusión/Fricción en el sistema sociofónico del castellano de Chile. Tesis de Magíster en Lingüística Aplicada, Concepción: Universidad de Concepción, 2011.

Giles, Howard. Patterns of evaluation in Reactions to R. P., South Welsh and Somerset accented speech. British Journal of Social and Clinical Psychology 10 (1971): 280281.

Hernández, Juan Manuel y Almeida, Manuel. Metodología de la investigación sociolinguística. Málaga: Editorial Comares, 2005.

Hernández-Campoy, Juan Manuel. El fenómeno de las actitudes y su medición en sociolingüística. Universidad de Murcia. (sf).

Hernández, Marisol y Dinamarca, Javiera. Actitudes lingüísticas hacia el mapudungun y el 
castellano: estudio exploratorio en dos comunidades pewenche del Alto Biobío. Nueva Revista del Pacífico 69 (2018): 51-66.

Hymes, Dell. Model of the Interaction of Language and Social Life. Directions in Sociolinguistics: The Ethnography of Communication. Ed. J. J. Gumperz y D. Hymes. Nueva York: Holt, Rinehart \& Winstor, 1974. 35-71.

Labov, William. Modelos sociolingüísticos. Madrid, España: Ediciones Cátedra, 1983.

Lenz, Rudolf. El español en Chile. Buenos Aires: Universidad de Buenos Aires, 1940. López, Humberto. Sociolingüística. Madrid: Gredos, 2004.

Moreno, Francisco. Metodología sociolingüística. Madrid, España: Ediciones Gredos, 1990.

Nietzsche, Friedrich. Así habló Zaratustra. Bogotá: Fonolibros, 2005.

Ortiz, Héctor y Saavedra, Eugenia. La fonética en Chile: Bibliografía analítica 1829-2000. Santiago: Phoné Libros, 2003.

Ramírez, Yesenia. Una aproximación a la complejidad del caudal léxico de profesores chilenos en formación: análisis cualitativo y cuantitativo de estructuras plurilexicales. Nueva Revista del Pacífico 71 (2019): 143-160.

Reyes, Leonora. La escuela en nuestras manos. Las experiencias educativas de la Asociación General de Profesores y la Federación Obrera de Chile (1921-1932). Santiago: Quimantú, 2014.

Sadowsky, Scott y Salamanca, Gastón. El inventario fonético del español de Chile: Principios orientadores, inventario provisorio de consonantes y sistema de representación (AFI-CL). Onomazein 24(2) (2011): 61-84.

Sadowsky, Scott. Variación sociofonética de las consonantes del español de Chile. Sociolinguistic studies 9(1) (2015): 71-92.

Salazar, Aura. El prestigio frente a la identidad: las actitudes lingüísticas de los monterianos hacia el español hablado en Montería. Cuadernos de Lingüística Hispánica 25 (2014): 39-55.

Serrano, María José. Sociolingüística. Barcelona: Ediciones del Serbal, 2011.

Silva, Carmen y Enrique, Andrés. Sociolinguiística y pragmática del español. Washington: Georgetown University Press, 2017.

Valdivieso, Humberto. El español culto y formal de Concepción. Revista de Linguiística teórica y Aplicada 16 (1978): 125-133.

Valdivieso, Humberto. Prestigio y estigmatización: factor determinante en la enseñanza institucionalizada de la lengua materna. Revista de Lingüística teórica y Aplicada 21(1983): 137-142. 